\title{
Methods in Molecular Biology
}

\section{Series Editor}

John M. Walker

School of Life Sciences

University of Hertfordshire

Hatfield, Hertfordshire, AL10 9AB, UK

For other titles published in this series, go to

www.springer.com/series/7651 


\title{
Cancer Gene Profiling
}

\author{
Methods and Protocols
}

Edited by

\section{Robert Grützmann and Christian Pilarsky}

Department of Surgery, University Hospital Carl Gustav Carus,

University Dresden, Dresden, Germany

浩: Humana Press 


\section{Editors}

Robert Grützmann

Department of Surgery

University Hospital Carl Gustav Carus

University Dresden

Dresden

Germany

Robert.Gruetzmann@uniklinikum-dresden.de
Christian Pilarsky

Department of Surgery

University Hospital Carl Gustav Carus

University Dresden

Dresden

Germany

christian.pilarsky@mailbox.tu-dresden.de
ISSN 1064-3745

ISBN 978-1-934115-76-3

DOI 10.1007/978-1-59745-545-9

Springer Dordrecht Heidelberg London New York
e-ISSN 1940-6029

e-ISBN 978-1-59745-545-9

Library of Congress Control Number: 2009930638

(C) Humana Press, a part of Springer Science+Business Media, LLC 2010

All rights reserved. This work may not be translated or copied in whole or in part without the written permission of the publisher (Humana Press, c/o Springer Science+Business Media, LLC, 233 Spring Street, New York, NY 10013, USA), except for brief excerpts in connection with reviews or scholarly analysis. Use in connection with any form of information storage and retrieval, electronic adaptation, computer software, or by similar or dissimilar methodology now known or hereafter developed is forbidden.

The use in this publication of trade names, trademarks, service marks, and similar terms, even if they are not identified as such, is not to be taken as an expression of opinion as to whether or not they are subject to proprietary rights. While the advice and information in this book are believed to be true and accurate at the date of going to press, neither the authors nor the editors nor the publisher can accept any legal responsibility for any errors or omissions that may be made. The publisher makes no warranty, express or implied, with respect to the material contained herein.

Printed on acid-free paper

Humana is part of Springer Science+Business Media (www.springer.com) 


\title{
Preface
}

\author{
Science is the agent between imagination and reality
}

\section{(Anonymous)}

During the last few years, the methods for analysing cancer-related genes on a molecular level have changed rapidly. With the advent of automated sequencing, new and faster investigations have become possible. This has led to the collection of a large number of DNA samples, such as Expressed Sequence Tag (EST) libraries whose entries run into millions. The advances in DNA sequencing technologies resulted in rapid improvements in oligonucleotide synthesising technologies, which has allowed researchers to produce oligonucleotides for each and every imaginable sequence at a low cost. Finally, the method of polymerase chain reaction (PCR), and other improvements in enzymatic in vitro amplification of nucleic acids, gave researchers the opportunity to use low amounts of nucleic acids for analysis. This enabled the research community to investigate the populations of cells in a given tissue.

Sometimes it takes only a few advances for a technology to be successful - for example, the concept of arraying biological probes in a reproducible manner, and the use of these arrays instead of a single probe, greatly advanced biomedical research, especially as it was discovered that everything is arrayable. It has also changed the landscape of science in another way: a reduction of costs. The costs of generating such an array are high, but the costs of replicating such arrays are not. This key fact has led to a growth in the number of biotech companies that design and produce arrays, and that today more and more researchers have access to and use. Such unrestricted access to these resources has really been the key to the biomedical research revolution we see today.

In this book, we have brought together the experiences of leading scientists in the discipline of cancer gene profiling. We have included several microarray techniques, as well as methods for arraying tissues and proteomics, because cancer genes can be profiled in different ways. Such different approaches are needed to understand the key stages of cancer development, because using only one technique would be insufficient. Therefore, we attempt to give an overview of the state-of-the-art methods that will enable the reader to perform these experiments successfully. It has been written for any student or practitioner with an interest in cancer gene profiling, and can be used in any well-equipped research laboratory. It may also serve as a demonstration of the kind of analysis that is possible today and will be complementary to other textbooks in the area of biomedical research.

This book has been divided into five main sections. The first section covers techniques to get clinically relevant cancer material through the best methods of sample collection and storage.

The second part begins with an overview of gene expression technology and gives an introduction to the latest cancer gene profiling technologies. Because cancer gene profiling is more then just the profiling of cancer gene expression, we have also included techniques for comparative genomic hybridisation (CGH) arraying and single-nucleotide polymorphism (SNP) analysis, and proteomic techniques. 
The third section contains real-life examples for the different technologies, and shows the full potential of cancer gene profiling today. This potential can only be utilised by the use of adequate bioinformatics tools. These tools are covered in the fourth part of the book. Because a cancer gene profiling experiment will most often lead to numerous candidate genes, which, in turn, have to be further validated and analysed, examples of performing post profiling experiments can be found in the final section of the book. It should be noted that all of the chapters in the book are linked by the description of particular successful experiments that were performed within the field of gene expression profiling.

We offer our gratitude to all of the contributing authors and the staff of Humana Press without their help, this book would not have been possible. We also thank our families for their love and patience. Finally, we are indebted to our mentor Hans Detlev Saeger for his unwavering support.

Science is not just a profession - it should also be fun. This fun comes from the inception of an idea that goes on to be proven through experimentation, or, as we found in a Chinese fortune cookie: "The impossible is only the untried."

We hope that you will not only be successful, but will also have fun using our book in your research.

Dresden, Germany 


\section{Contents}

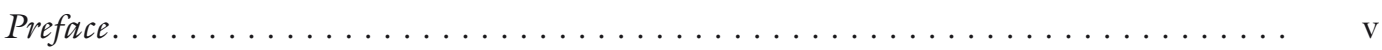

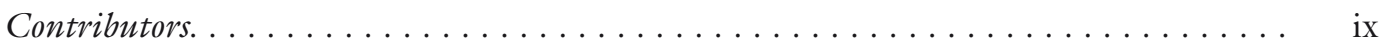

1. Organizational Issues in Providing High-Quality Human Tissues and Clinical Information for the Support of Biomedical Research. . . . . . . . . I 1

Walter C. Bell, Katherine C. Sexton, and William E. Grizzle

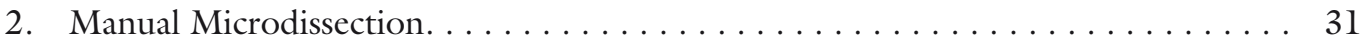

Glen Kristiansen

3. Laser Microdissection . . . . . . . . . . . . . . . . . . . . 39

Anja Rabien

4. Tissue Microarrays. . . . . . . . . . . . . . . . . . . . . . . . 49

Ana-Maria Dancau, Ronald Simon, Martina Mirlacher,

and Guido Sauter

5. A Decade of Cancer Gene Profiling: From Molecular

Portraits to Molecular Function. . . . . . . . . . . . . . . . . . . . . . 61

Henri Sara, Olli Kallioniemi, and Matthias Nees

6. Mining Expressed Sequence Tag (EST)Libraries

for Cancer-Associated Genes . . . . . . . . . . . . . . . . . . . . . . . . 89

Armin O. Schmitt

7. Automated Fluorescent Differential Display for Cancer Gene Profiling . . . . . . . 99

Jonathan D. Meade, Yong-jig Cho, Blake R. Shester, Jamie C. Walden, Zhen Guo, and Peng Liang

8. Manual Microdissection Combined with Antisense

RNA-LongSAGE for the Analysis of Limited Cell Numbers . . . . . . . . . . . . 135

Jutta Lüttges, Stephan A. Habn, and Anna M. Heidenblut

9. Quantitative DNA Methylation Profiling on a High-Density

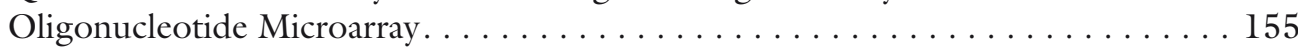

Anne Fassbender, Jörn Lewin, Thomas König, Tamas Rujan,

Cecile Pelet, Ralf Lesche, Jürgen Distler, and Matthias Schuster

10. Single-Nucleotide Polymorphism (SNP) Analysis

to Associate Cancer Risk . . . . . . . . . . . . . . . . . . . . . . . . . . . . . . . . . . . . . 171

Julie Earl and William Greenhalf

11. Application of Proteomics in Cancer Gene Profiling:

Two-Dimensional Difference in Gel Electrophoresis (2D-DIGE) . . . . . . . . . . . 197

Deepak Haribaran, Mark E. Weeks, and Tatjana Crnogorac-Jurcevic

12. Search for and Identification of Novel Tumor-Associated Autoantigens . . . . . . . 213

Karsten Conrad, Holger Bartsch, Ulvich Canzler, Christian Pilarsky,

Robert Grïtzmann, and Michael Bachmann 
13. Integrative Oncogenomic Analysis of Microarray

Data in Hematologic Malignancies

Jose A Martinez-Climent, Lorena Fontan, Vicente Fresquet,

Eloy Robles, Maria Ortiz, and Angel Rubio

14. Cancer Gene Profiling in Pancreatic Cancer. . . . . . . . . . . . . . . . . . . . . . 279

Felip Vilardell and Christine A. Iacobuzio-Donabue

15. Cancer Gene Profiling in Prostate Cancer . . . . . . . . . . . . . . . . . . . 293 Adam Foye and Phillip G. Febbo

16. Cancer Gene Profiling for Response Prediction

B. Michael Ghadimi and Marian Grade

17. The EGFR Pathway as an Example for Genotype:

Phenotype Correlation in Tumor Genes.

Ulrike Mogck, Evay Goekkurt, and Jan Stoeblmacher

18. Quantitation Of CD39 Gene Expression in Pancreatic Tissue

by Real-Time Polymerase Chain Reaction

Martin Loos, Beat Künzli, and Helmut Friess

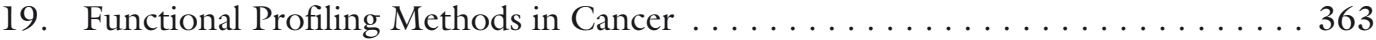

Joaquin Dopazo

20. Calibration of Microarray Gene-Expression Data . . . . . . . . . . . . . 375

Hans Binder, Stephan Preibisch, and Hilmar Berger

21. Meta-analysis of Cancer Gene-Profiling Data. . . . . . . . . . . . . . . . . . . . . 409

Xinan Yang and Xiao Sun

22. Target Gene Discovery for Novel Therapeutic

Agents in Cancer Treatment . . . . . . . . . . . . . . . . . . . . . . . . . . 427

Ole Ammerpobl, Sanjay Tiwari, and Holger Kalthoff

Index. . . . . . . . . . . . . . . . . . . . . . . . . . . . . . . . . . . . . . 447 


\section{Contributors}

Ole Ammerpohl - Clinic for General Surgery and Thoracic Surgery, Division Molecular Oncology, University Hospital of Schleswig, Kiel, Germany

Michael Bachmann - Institute for Immunology, Technical University Dresden, Dresden, Germany

Holger Bartsch - Institute for Immunology, Technical University Dresden, Dresden, Germany

Walter C. Bell • Department of Pathology, University of Alabama at Birmingham, Birmingham, AL, USA

Hilmar Berger - Institute for Medical Informatics, Statistics and Epidemiology, University of Leipzig, Leipzig, Germany

Hans Binder - Interdisciplinary Centre for Bioinformatics, University of Leipzig, Leipzig, Germany

Ulrich CAnZler • Institute for Immunology, Technical University Dresden, Dresden, Germany

Yong-JIG CHO • Department of Cell Biology, Vanderbilt-Ingram Cancer Center, School of Medicine, Vanderbilt University, Nashville, TN, USA

Karsten Conrad - Institute for Immunology, Technical University Dresden, Dresden, Germany

Tatjana Crnogorac-Jurcevic - Cancer Research UK Molecular Oncology Unit, Barts and The London Queen Mary's School of Medicine and Dentistry, John Vane Science Centre, London, UK

ANa-Maria Dancau • Institute of Pathology, University of Hamburg, Hamburg, Germany

Jürgen Distler • Science Department, Epigenomics AG, Berlin, Germany

Joaquín Dopazo • Bioinformatics Department, Centro de Investigación Príncipe Felipe, Valencio, Spain

Julie EARL • Division of Surgery and Oncology, University of Liverpool, Liverpool, UK

Anne Fassbender • Science Department, Epigenomics AG, Berlin, Germany

Phillip G. Fевво - Departments of Medicine and Molecular Genetics and Microbiology, Duke Institute for Genome Science and Policy, Duke University, Durham, NC, USA

Lorena FonTan - Division of Oncology, Center for Applied Medical Research, University of Navarra, Pamplona, Spain

Adam Foye - Departments of Medicine and Molecular Genetics and Microbiology, Duke Institute for Genome Science and Policy, Duke University, Durham, NC, USA

Vicente FresQuet - Division of Oncology, Center for Applied Medical Research, University of Navarra, Pamplona, Spain 
Helmut Friess • Department of Surgery, Technische Universität München, Munich, Germany

B. Michael GHadimi - Department of General and Visceral Surgery, University Medical Center Göttingen, Georg-August-University, Göttingen, Germany

Eray GoekKurT • Department of Internal Medicine I, University Hospital Carl Gustav Carus, University Dresden, Dresden, Germany

MARIAN GRADE - Department of General and Visceral Surgery, University Medical Center Göttingen, Georg-August-University, Göttingen, Germany

William Greenhalf • Division of Surgery and Oncology, University of Liverpool, Liverpool, UK

William E. GRIzZle - Department of Pathology, University of Alabama at Birmingham, Zeigler Research Building, Birmingham, AL, USA

Robert Grützmann • Department of Surgery, University Hospital Carl Gustav Carus, University Dresden, Dresden, Germany

ZHeN GuO • GenHunter Corporation, Nashville, TN, USA

Stephan A. Hahn • Molecular GI-Oncology (MGO), Center for Clinical Research (ZKF), Ruhr-University Bochum, Bochum, Germany

Deepak Hariharan - Cancer Research UK Molecular Oncology Unit, Barts and The London Queen Mary's School of Medicine and Dentistry, John Vane Science Centre, London, UK

Anna M. Heidenblut • Molecular GI-Oncology (MGO), Center for Clinical Research (ZKF), Ruhr-University Bochum, Bochum, Germnay

Christine A. Iacobuzio-Donahue - Department of Pathology, GI/Liver Division, Johns Hopkins Medical Institutions, The Sol Goldman Pancreatic Cancer Research Center, Baltimore, MD, USA

Olli Kallioniemi - VTT Medical Biotechnology, Turku, Finland

Holger KalthOFF • Clinic for General Surgery and Thoracic Surgery, Division Molecular Oncology, University Hospital of Schleswig-Holstein, Kiel, Germany

Thomas König • Science Department, Epigenomics AG, Berlin, Germany

Glen Kristiansen - Department of Pathology, University Hospital Zurich, Zurich, Switzerland

Beat KünZli • Department of Surgery, Technische Universität München, Munich, Germany

Ralf Lesche • Science Department, Epigenomics AG, Berlin, Germany

Jörn LewIN • Science Department, Epigenomics AG, Berlin, Germany

Peng LiAng - Department of Cell Biology, Vanderbilt-Ingram Cancer Center, School of Medicine, Vanderbilt University, Nashville, TN, USA

Martin Loos - Department of Surgery, Technische Universität München, Munich, Germany

JuTTA LÜTTGES • Institute für Pathology, Saarbrücken Hospital, Saarbrücken, Germany

Jose A. Martínez-Climent • Division of Oncology, Center for Applied Medical Research, University of Navarra, Pamplona, Spain 
Jonathan D. Meade • GenHunter Corporation, Nashville, TN, USA

Martina Mirlacher - Institute of Pathology, University of Hamburg, Hamburg, Germany

Ulrike Mogck • Department of Internal Medicine I, University Hospital Carl Gustav Carus, University Dresden, Dresden, Germany

Matthias Nees • VTT Medical Biotechnology, Turku, Finland

María Ortiz - CEIT and TECNUN, University of Navarra, San Sebastián, Spain

Cecile Pelet - Science Department, Epigenomics AG, Berlin, Germany

Christian Pilarsky - Department of Surgery, University Hospital Carl Gustav Carus, University Dresden, Dresden, Germany

Stephan Preibisch - Max-Planck-Institute for Molecular Cell Biology and Genetics, Dresden, Dresden, Germany

Anja Rabien - Research Division, Department of Urology, Charité - Universitätsmedizin Berlin, Campus Charité Mitte, Berlin, Germany

Eloy Robles - Division of Oncology, Center for Applied Medical Research, University of Navarra, Pamplona, Spain

Angel Rubio - CEIT and TECNUN, University of Navarra, San Sebastián, Spain

Tamas Rujan - Science Department, Epigenomics AG, Berlin, Germany

Henri Sara - VTT Medical Biotechnology, Turku, Finland

Guido SAUTER - Institute of Pathology, University of Hamburg, Hamburg, Germany

Armin O. Schmitт • Institute for Animal Sciences, Humboldt-Universität zu Berlin, Berlin, Germany

Matthias Schuster - Science Department, Epigenomics AG, Berlin, Germany

Katherine C. SeXton - Comprehensive Cancer Center, University of Alabama at Birmingham, Birmingham, AL, USA

Blake R. Shester • GenHunter Corporation, Nashville, TN, USA

Ronald SimOn - Institute of Pathology, University of Hamburg, Hamburg, Germany

Jan Stoehlmacher • Department of Internal Medicine I, University Hospital Carl

Gustav Carus, University Dresden, Dresden, Germany

XIAO Sun - Division of Bioinformatics, State Key Laboratory of Bioelectronics (ChienShiung Wu Laboratory), Southeast University, Nanjing, China

SAnjay TiWARI - Division Molecular Oncology, Clinic for General Surgery and Thoracic Surgery, University Hospital of Schleswig-Holstein, Kiel, Germany

Felip Vilardell • Department of Pathology, GI/Liver Division, Johns Hopkins

Medical Institutions, The Sol Goldman Pancreatic Cancer Research Center,

Baltimore, MD, USA

Jamie C. Walden - GenHunter Corporation, Nashville, TN, USA

Mark E. Weeks - Cancer Research UK Molecular Oncology Unit, Barts and The

London Queen Mary's School of Medicine and Dentistry, John Vane Science Centre, London, UK

XINAN YANG - Division of Bioinformatics, State Key Laboratory of Bioelectronics, (Chien-Shiung Wu Laboratory), Southeast University, Nanjing, Nanjing, China 